\title{
Neonatal Umbilical Arterial Catheter Removal Is Accompanied by a Marked Decline in Phlebotomy Blood Loss
}

\author{
Patrick D. Carroll ${ }^{a}$ M. Bridget Zimmerman ${ }^{b}$ Demet Nalbant ${ }^{c}$ Earl L. Gingerich ${ }^{c}$ \\ Guohua An $^{d} \quad$ Gretchen A. Cress $^{c}$ Peter Veng-Pedersen ${ }^{d} \quad$ John A. Widness ${ }^{c}$ \\ a'Women and Newborn Clinical Program, Intermountain Healthcare, Dixie Regional Medical Center, St. George, UT, \\ USA; ${ }^{b}$ Department of Biostatistics, College of Public Health, The University of lowa, lowa City, IA, USA; ' Stead Family \\ Department of Pediatrics, Roy J. and Lucille A. Carver College of Medicine, The University of lowa, lowa City, IA, USA; \\ ${ }^{\mathrm{d}}$ Department of Pharmaceutical Sciences and Experimental Therapeutics, University of lowa College of Pharmacy, \\ lowa City, IA, USA
}

\section{Keywords}

Phlebotomy blood loss - Umbilical arterial catheter .

Anemia of prematurity

\section{Abstract}

Background: Umbilical arterial catheters (UACS) are frequently used in critically ill neonates. UAC are convenient, reliable, and allow for caregiver convenience in performing painless arterial blood sampling. We hypothesized that UAC removal in extremely low birth weight (ELBW) neonates will result in significantly less phlebotomy blood loss (PBL) after correcting for severity of illness. Study Design and Methods: PBL was measured at a single center in 99 ELBW infants who survived to day 28. Individual infant's paired daily PBL for the two 24-h periods before and after UAC removal were compared using the paired $t$ test. Daily PBL up to 7 days before and 7 days after UAC removal were compared using a logistic regression with mixed model analysis for repeated measures. Cumulative 28-day phlebotomy loss was evaluated by multiple linear regression analysis. Results: PBL $24 \mathrm{~h}$ before and after UAC removal were $1.7 \mathrm{~mL}(95 \% \mathrm{Cl} 1.5-1.9)$ and 0.9 $\mathrm{mL}(95 \% \mathrm{Cl} 0.8-1.0 ; p<0.0001)$, respectively. Cumulative 28day PBL increased by $2.2 \mathrm{~mL}( \pm 0.7)$ per day that a UAC was present with or without correction for severity of illness ( $p<$ $0.001)$. Conclusion: UAC removal is independently associated with a marked decline in PBL. We speculate the ease and convenience of UAC blood sampling lead to more frequent blood testing and greater PBL.

(c) 2020 S. Karger AG, Basel

\section{Introduction}

Umbilical arterial catheters (UACs) are commonly used in the care of critically ill neonates. The presence of a UAC allows for easy, convenient access of direct arterial blood sampling and continuous blood pressure monitoring without the pain of heel stick capillary blood sampling. Despite these advantages, UACs carry the risk of thrombus formation, hypertension, central line-associated blood stream infections, and decreased cerebral perfusion during sampling [1-4]. Because of the ease of UAC use and its convenience for blood sampling, they have the potential to lead to unnecessary, more frequent blood sampling leading to increased phlebotomy blood loss (PBL). 
Increased PBL results in greater rates and volumes of red blood cell (RBC) transfusion [5]. The increase in PBL being reported associated with more frequent transfusions in neonates is of concern because of the association of transfusion with intraventricular hemorrhage [6], necrotizing enterocolitis [7], bronchopulmonary dysplasia [8], and increased mortality [9].

A better understanding of the impact of UAC use may help clinicians decrease the rate of PBL in infants, particularly in extremely low birth weight (ELBW) infants. It is uncertain whether the higher PBL is due to increased severity of illness or the presence of a UAC. Thus, our primary study objective was to evaluate whether the anticipated gradual decline in PBL is abruptly accelerated immediately following UAC removal. While it is reasonable to anticipate that the presence of UAC may encourage physicians to order more frequent laboratory testing leading to increased PBL, we are unaware of a prior study having investigated this. In the present study, we hypothesized that UAC removal in ELBW neonates will result in significantly less PBL after correcting for severity of illness.

\section{Materials and Methods}

IRB approval was obtained, and written informed consent was provided by all parents or guardians; this included consent for the use of data in future studies. Enrollment was between April 2007 and December 2016 with all neonates having been previously reported in convenience enrollment studies [10-14]. Neonates with birth weight $<1,000 \mathrm{~g}$ who were intubated and survived to 28 days were included. Neonates excluded were those presenting with hematologic diseases (except for anemia of prematurity), receiving $\mathrm{RBC}$ transfusions before enrollment, or receiving erythropoiesisstimulating agents. UAC placement was not required for inclusion.

Throughout the study period, the clinical management of infants in our single-center NICU did not undergo identifiable, impactful practice changes. Throughout the decade of study, subjects were managed in a coordinated fashion with changes made primarily on twice daily rounds by a team of physicians, nurses, nutritionists, and pharmacists that changed every 2 weeks. During the study period, there were few changes in staff physician personnel, with a total of 8-10 supervising faculty neonatologists. With the exception of RBC transfusion decisions [15] and the practice of placing UAC in all infants with birth weights $<1.5 \mathrm{~kg}$ requiring mechanical ventilation during the first day of life, clinical blood management decisions were not dictated by specific protocols or guidelines. Such decisions included frequency of performing specific blood testing as dictated by the clinical stability of individual infants and the timing of catheter removal. Unit standard practice was to remove all central lines as soon as possible to avoid central line-associated blood stream infections. No other specific UAC intervention policy was introduced during the study period. During the study period, catheter and peripheral blood sampling required
Table 1. Neonatal demographic data $(n=99)$
Birth weight (mean $+\mathrm{SD}), \mathrm{kg}$

Gestational age (mean $+\mathrm{SD})$, weeks

Gender, \% male

Prenatal steroids, \% received

Mode of delivery, \% cesarean section

Median Apgar score (25-75)

$1-\min$

5 - $\min$

$0.726(+0.153)$
$254 / 7(+14 / 7)$
47
96
67

$4(3-6)$
$7(6-8)$

identical blood volumes, and there were no laboratory instrumentation changes requiring changes in blood volumes needed for commonly performed NICU laboratory blood tests.

This was a retrospective observational study using prospectively collected PBL data. All ELBW study infants included survived to 28 days. Accurate PBL volumes were determined by weighing each sample before laboratory analysis as previously described [12]. Birth weight was used for values reported in milliliters per kilogram. Each blood sample was recorded including the type of collection tube used, laboratory testing performed, and residual blood remaining after laboratory analysis.

PBL with a UAC included any blood loss that occurred while a UAC was present. Similarly, PBL without a UAC included any blood loss that occurred following UAC removal. Cumulative PBL for the 28-day study period was calculated by summing the PBL for each day. The number of ELBW infants with a UAC was initially large and gradually declined as UACs were removed.

The presence of and the precise times of UAC removal were determined from the medical health record. Based on the NICU practice, we assumed that UAC removal represented the time point at which the clinical treatment team had determined the patient to be stable enough to permit UAC removal. PBL was evaluated for up to seven 24 -h periods before and for seven 24 -h periods after UAC removal. This method was used to compare patients with more similar severity of illness. Only full 24 -h periods were included in this analysis. Because PBL is predictably high in the first 2 days of life [14], PBL during these days were not included in this specific analysis to avoid falsely elevating the PBL and overestimating the effect of the UAC in comparing PBL with and without a UAC.

Score for neonatal acute physiology (SNAP) was determined as an indicator for severity of illness in neonates at birth and during the two 24-h periods before and after UAC removal $[16,17]$. Because once the UAC was removed, the $\mathrm{PaO}_{2} \mathrm{SNAP}$ component was no longer available for inclusion, a modified SNAP score was used for comparing the 24-h periods before and after UAC removal.

\section{Statistics}

PBL was compared for the $24 \mathrm{~h}$ before and after UAC removal using the paired $t$ test. Regression analysis following logarithmic transformation to normalize the distribution of $\mathrm{PBL}$ data was also performed. PBL for the UAC and no-UAC periods were compared using a logistic regression with mixed-model analysis for repeated measures. This accounted for the fixed and random effects within and between study subjects. Natural logarithmic transformation was also applied to the data to normalize the PBL data distribution. Linear trends were compared for up to 7 days before and 7 days after UAC removal. Multiple linear regression analysis was used to 


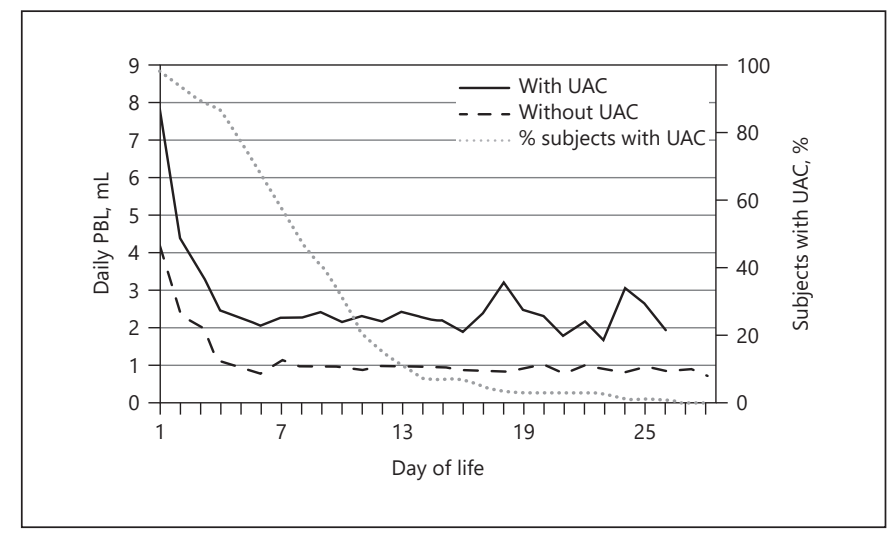

Fig. 1. Daily PBL is higher among subjects with a UAC present for each of the first 28 days.

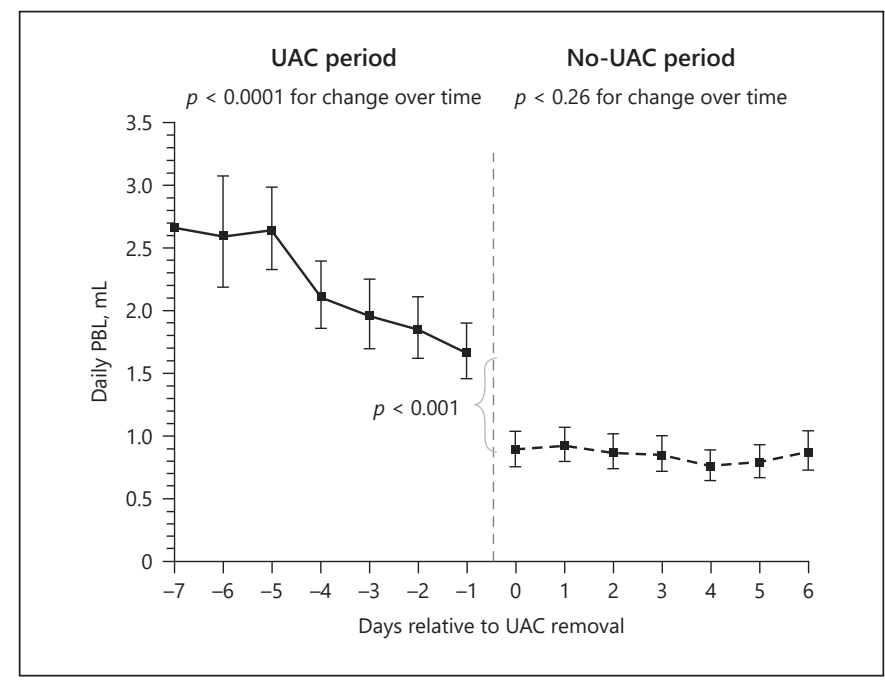

Fig. 3. Abrupt decrease in PBL after UAC removal. A gradual significant decline in PBL was observed until UAC removal occurred $(p=0.0001)$. After UAC removal, there was an abrupt decrease in PBL $(p<0.001)$ from the UAC to the no-UAC period followed by a stable rate of change in daily $\mathrm{PBL}(p=0.26)$.

evaluate the impact of birth weight, SNAP score, and UAC days on 28-day cumulative PBL. The interaction effect of UAC in days to the nearest tenth of a day and SNAP score at birth was also evaluated using this regression analysis.

\section{Results}

Demographic data for the 99 ELBW study infants are presented in Table 1 . The average daily PBL with a UAC in place was significantly higher than the average daily PBL without a UAC in place for each day from days 2 to $22(p<0.05)$ but not statistically different for days 1 and

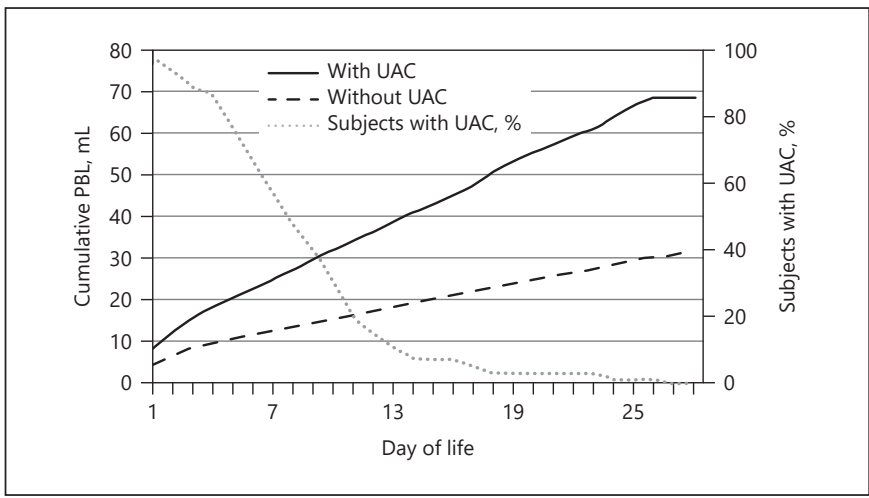

Fig. 2. Cumulative 28-day $\mathrm{PBL}$ is higher among subjects with a UAC present $(p<0.001)$.

23-28 (Fig. 1). There was a rapid decline in daily PBL over the first 2-3 days of life in subjects both with and without a UAC. The average cumulative 28 -day PBL with a UAC in place was $69 \mathrm{~mL}(108 \mathrm{~mL} / \mathrm{kg})$ while the average cumulative PBL without a UAC in place was $32 \mathrm{~mL}(43 \mathrm{~mL} / \mathrm{kg}$; $p<0.001$ ) (Fig. 2). The average cumulative number of heel stick punctures per neonate on day 28 was 60 (range 10-133), and the average cumulative number of independent blood draws (UAC blood draws plus heel stick puncture) on day 28 was 125 (range 41-268). The average cumulative number of independent blood draws on day 28 during the time when a UAC was in place was 179 while the average cumulative number of independent blood draws during the period without a UAC was $104(p<$ $0.001)$.

Daily PBL progressively decreased during the 7 days prior to UAC removal but abruptly dropped immediately following UAC removal $(p<0.001)$ and remained constant for the next 7 days (Fig. 3). There was a significant decreasing linear trend in mean PBL in the 7-day period before UAC removal $(p<0.0001)$. In contrast, no significant linear trend in PBL was observed during the 7 days after UAC removal $(p=0.26)$. During the 24 -h period before UAC removal, the mean PBL was $1.7 \mathrm{~mL}$ (95\% CI $1.5-1.9)$, compared to $0.9 \mathrm{~mL}$ (95\% CI $0.8-1.0) 24 \mathrm{~h}$ after UAC removal rather than the expected PBL of $1.5 \mathrm{~mL}$ if the declining trend had continued. The average number of $\mathrm{RBC}$ transfusions on day 28 during the time when a UAC in place was 5.7 while the average number of RBC transfusions during the period without a UAC was 2.0. $(p=0.002)$.

Modified SNAP score on the day before UAC removal indicated a significantly lower $(p=0.03)$ severity of illness of $6.2( \pm 3.9)$ compared to the day after UAC removal, 7.3 


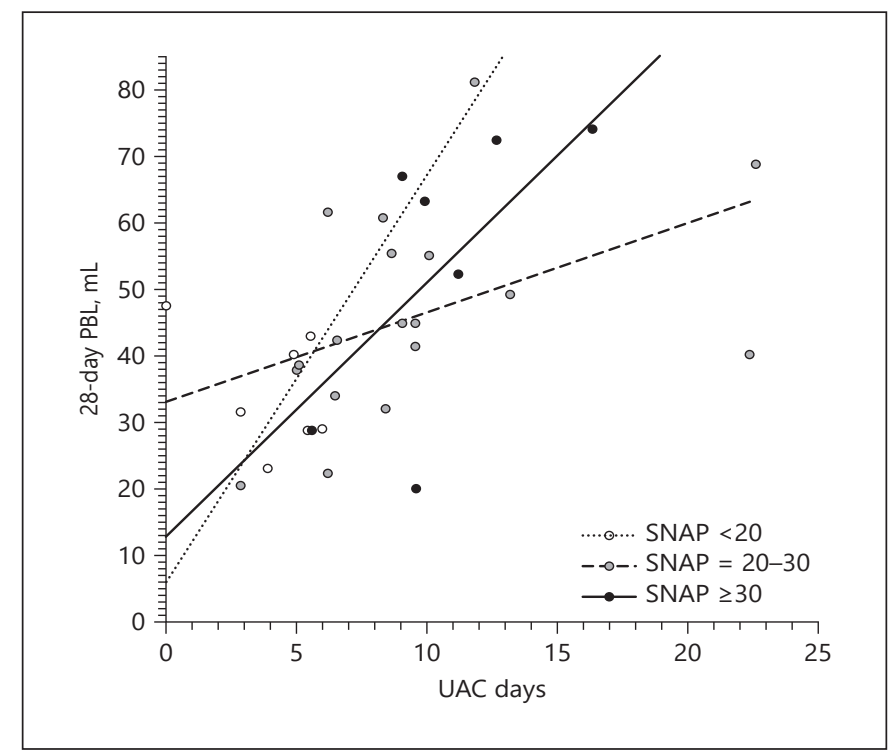

Fig. 4. The interaction of SNAP score at birth and the UAC days are indicated by different SNAP scores having different 28-day PBL. Modeled UAC days increased the predicted PBL by $6.9 \mathrm{~mL}$ for each day a UAC was present when the SNAP score was $<20$ at birth. PBL increased by $3.9 \mathrm{~mL}$ for each day a UAC was present when the SNAP score was $\geq 30$ at birth. However, when the SNAP score at birth was 20-29, the predicted 28-day PBL only increased by $1.2 \mathrm{~mL}$.

( \pm 3.7$)$. Factors leading to higher post-removal modified SNAP scores included low blood pressure, abnormal respiratory rate, and high heart rate. Regression modeling demonstrated a 92\% higher PBL (95\% CI 61-130\%) during the 24-h period before UAC removal compared to that after removal $(p<0.0001)$. After adjusting for modified SNAP score, the estimated mean PBL was $102 \%$ greater (95\% CI: 64-149\%) during the 24 -h period before UAC removal than after UAC removal $(p<0.0001)$.

We used multiple linear regression analysis to estimate the 28-day cumulative PBL. Analysis with stepwise elimination of nonsignificant cofactors indicated that only birth weight and UAC days remained significant predictors of 28-day cumulative PBL. SNAP score at birth was not a significant predictor of PBL in this model, which demonstrated that for every day a UAC is present, the cumulative 28-day PBL increases by $2.2 \mathrm{~mL}(p<0.0001)$ and that cumulative 28 -day PBL decreases by $2.8 \mathrm{~mL}$ for every $100 \mathrm{~g}$ of birth weight $(p=0.004)$. There was no difference in UAC days between the first (6.9) and second (8.0) half of the 10-year study period $(p=0.23)$.

An interaction effect was observed between SNAP score at birth and UAC days. The effect that UAC days had on 28-day cumulative PBL varied based on the birth
SNAP score (Fig. 4). The 28-day PBL increased by 6.9, 1.2, and $3.9 \mathrm{~mL}$ for each UAC day when the initial SNAP score was $<20(p=0.002), 20-29(p=0.02)$, and $\geq 30(p<$ 0.0001 ), respectively. Inclusion of the interaction between SNAP score and UAC days improved the predictive value of this regression analysis $\left(R^{2}=0.618\right)$.

\section{Discussion}

The purpose of this study was to evaluate the effect of a UAC on PBL following its removal. Indeed, our findings demonstrate that an important factor leading to $\mathrm{PBL}$ is the presence of a UAC. We report a significantly greater PBL during the study periods with than without a UAC. Given the $<1-\mathrm{kg}$ birth weight of infants in this study, a PBL of 32 $\mathrm{mL}$ is equivalent to over $40 \mathrm{~mL} / \mathrm{kg}$ in the first 4 weeks of life, or approximately one-half of an infant's blood volume. After the high PBL in the first days of life, daily PBL among those without a UAC remained at approximately $1 \mathrm{~mL}$ PBL per day through day 28. This indicates that laboratory evaluation did not decrease in weeks 2,3 , or 4 as one might have expected as most infants' degree of illness lessened. Thus, in addition to decreasing PBL by limiting the use of UAC, it also appears there is an opportunity to critically evaluate the frequency and need of ongoing PBL during the first 28 days of life. A focus on both factors could result in lower PBL without compromising clinical care while also mitigating risks of anemia and $\mathrm{RBC}$ transfusion.

Smaller and more critically ill infants typically have higher PBL [18]. There is a direct relationship between the volume of blood removed for laboratory testing and that transfused into very low birth weight infants with correlation coefficients of $0.75-0.90[5,15,19,20]$. Furthermore, infants with UAC are generally more critically ill and therefore require more laboratory testing. While variation in PBL may be explained by differences in gestational age, birth weight, severity of illness, and other factors such as the presence of a UAC, the wide variations observed indicates considerable opportunity to limit PBL.

We accounted for the anticipated variation in PBL as a function of severity of illness in our statistical approach. To do so, we compared PBL for the 7 days before UAC removal with PBL for the 7 days after UAC removal. We also used regression modeling including SNAP scores.

The presence of a UAC had a greater impact on 28-day PBL among infants with the lowest SNAP scores, i.e., $<20$. This supports the principle of therapeutic inertia because a low clinical severity of illness may not have supported the degree of PBL, whereas the presence of a UAC may 
have influenced the decision to order additional laboratory analysis.

There are several possibilities that may explain greater $\mathrm{PBL}$ when a UAC is present. First, the presence of a UAC enables PBL to be easily obtained without pain. Several recent studies that were published after data had been gathered on the present study have demonstrated an association between brain injury in preterm infants and repeated procedural pain $[21,22]$. If these findings and their possible functional consequences become well established in the future, this may influence the decision of the provider regarding timing of UAC removal. We speculate that the elimination of pain leads to lower emotional distress when ordering laboratory blood testing. Second, therapeutic inertia is the principle by which a higher degree of medical intervention leads the caregivers to believe they are caring for more critically ill patients who require greater medical intervention [23]. Thus, the presence of a UAC may lead clinicians to believe the patient is critically ill. Furthermore, while the purpose of this study was not to evaluate the behavioral effects of individual caregivers, we speculate that the presence of a UAC may lead some caregivers to order blood tests that they might not order with no UAC present. This may explain in part the higher PBL when a UAC is present [24].

The mean duration of UAC presence of 7.5 days $( \pm 4.7)$ observed in this study is longer than the 5 days recommended by the Centers for Disease Control and Prevention [25-27]. It is unclear how closely these guidelines are followed in clinical practice, particularly among neonates at the limits of viability. However, the days of UAC use observed are consistent with other recent reports $[1,28]$. Although catheter use was not prescribed in the present study, the variation in UAC days had the advantage of offering more robust statistical modeling to address our questions.

There are several strengths of this study. One is the rigorous way PBL was determined, with $>95 \%$ of all blood sampling reported. This contrasts with some studies where PBL was estimated based on sample volumes required for laboratory tests multiplied by the number of tests performed.

We acknowledge several limitations of this study. First, randomization of ELBW infants to placement or no placement of UAC would be a more ideal study design. However, this is unfeasible and unethical. Alternatively, comparison to a control group that did not have a UAC placed even when indicated would be valuable. Our data set included 2 such infants who were part of this study. Second, compared to this single- center study, UAC placement and removal practices and phlebotomy practices may differ relative to other centers. For example, similar, singlecenter data from Europe published by Counsilman et al. [29] demonstrated significantly lower PBL (reported as $\mathrm{mL} / \mathrm{kg}$ of birth weight). However, our study cohort was approximately $100 \mathrm{~g}$ lighter at birth, 1 week less mature, and included only neonates requiring mechanical ventilation (compared to $75 \%$ requiring mechanical ventilation in the study by Counsilman et al. [29]). We previously reported that among our first 26 neonates enrolled for this study, only $33 \%$ of the blood volumes withdrawn were required by the laboratory instruments, while $59 \%$ were discarded as waste, and $8 \%$ were attributable to hidden blood loss in syringes, gauze pads, etc. [14].

In summary, UAC removal was associated with a marked decline in PBL in ELBW infants during the neonatal period. This decline in PBL was greater than anticipated by tracking the daily PBL before and after UAC removal. This finding should serve as a reminder to evaluate only essential blood testing needs when a UAC is present. We also advocate for developing consistent approaches to UAC placement and removal, and phlebotomy practices. Regarding the latter, consideration should include a more detailed evaluation of the necessity and frequency of specific blood testing, whether these were scheduled orders on a daily or weekly basis, and whether all were deemed necessary. Clearly, greater attention should be given to laboratory ordering practices especially when a UAC is present. Such efforts will likely lead to decreased PBL that will lead to decreased frequency of anemia and decreased need for RBC transfusion.

\section{Acknowledgments}

We acknowledge the contributions of Iowa's neonatal nurse research team in their interactions with the study subjects and their families. The neonatal nurses are partially supported by the NIH CTSA program grant UL1TR002537. We also acknowledge the monumental efforts and contribution of the laboratory phlebotomists who weighed and recorded all post-analysis blood samples. We would like to thank Robert D. Christensen and Ronald G. Strauss for their review of this manuscript prior to submission. Mark Hart provided valuable secretarial and editorial assistance. Finally, we acknowledge the families who trusted us to allow their children to participate in this study.

\section{Statement of Ethics}

IRB approval was obtained, and written informed consent was provided by all parents or guardians. 


\section{Disclosure Statement}

The authors declare that they have no conflicts of interest.

\section{Funding Sources}

J.A.W. received support for this work from NIH NHLBI Program Project Grant P01 HL046925. The neonatal nurses are partially supported by the NIH CTSA program grant UL1TR002537.

\section{Author Contributions}

Substantial contributions to conception and design, acquisition of data, or analysis and interpretation of data: P.D.C., B.Z., D.N., E.L.G., G.A., G.A.C., P.V.-P., J.A.W. Drafting the article or revising it critically for important intellectual content: P.D.C., B.Z., G.A., G.A.C., P.V.-P., and J.A.W. Final approval of the version to be published: P.D.C., D.N., E.L.G., G.A., G.A.C., P.V.-P., and J.A.W.

\section{References}

1 Elboraee MS, Toye J, Ye XY, Shah PS, Aziz K; Canadian Neonatal Network Investigators. Association between Umbilical Catheters and Neonatal Outcomes in Extremely Preterm Infants. Am J Perinatol. 2018 Feb;35(3):233-41.

2 McAdams RM, Winter VT, McCurnin DC, Coalson JJ. Complications of umbilical artery catheterization in a model of extreme prematurity. J Perinatol. 2009 Oct;29(10):685-92.

3 Ergaz Z, Simanovsky N, Rozovsky K, Leil SA, Ofek-Shlomai N, Revel-Vilk S, et al. Clinical outcome of umbilical artery catheter-related thrombosis - a cohort study. J Perinatol. 2012 Dec;32(12):933-40.

4 Mintzer JP, Parvez B, La Gamma EF. Umbilical arterial blood sampling alters cerebral tissue oxygenation in very low birth weight neonates. J Pediatr. 2015 Nov; 167(5):1013-7.

5 Valieva OA, Strandjord TP, Mayock DE, Juul SE: Effects of transfusions in extremely low birth weight infants: a retrospective study. J Pediatr 2009 Sep;155(3):331-7.e1.

6 Christensen RD, Baer VL, Lambert DK, Ilstrup SJ, Eggert LD, Henry E. Association, among very-low-birthweight neonates, between red blood cell transfusions in the week after birth and severe intraventricular hemorrhage. Transfusion. 2014 Jan;54(1):104-8.

7 La Gamma EF, Blau J. Transfusion-related acute gut injury: feeding, flora, flow, and barrier defense. Semin Perinatol. 2012 Aug; 36(4):294-305.

8 Zhang Z, Huang X, Lu H. Association between red blood cell transfusion and bronchopulmonary dysplasia in preterm infants. Sci Rep. 2014 Mar;4(1):4340.

9 dos Santos AM, Guinsburg R, de Almeida MF, Procianoy RS, Leone CR, Marba ST, et al. Red blood cell transfusions are independently associated with intra-hospital mortality in very low birth weight preterm infants. J Pediatr 2011;159:371-6.e1-3.

10 Nalbant D, Bhandary P, Matthews NI, Schmidt RL, Bogusiewicz A, Cress GA, et al. Comparison of multiple red cell volume methods performed concurrently in premature infants following allogeneic transfusion. Pediatr Res. 2013 Nov;74(5):592-600.
11 Schmidt RL, Mock DM, Franco RS, Cohen RM, North AK, Cancelas JA, et al. Antibodies to biotinylated red blood cells in adults and infants: improved detection, partial characterization, and dependence on red blood cellbiotin dose. Transfusion. 2017 Jun;57(6): 1488-96.

12 Freise KJ, Widness JA, Veng-Pedersen P. Erythropoietic response to endogenous erythropoietin in premature very low birth weight infants. J Pharmacol Exp Ther. 2010 Jan;332(1):229-37.

13 Arthur CM, Nalbant D, Feldman HA, Saeedi BJ, Matthews J, Robinson BS, et al. Anemia induces gut inflammation and injury in an animal model of preterm infants. Transfusion. 2019 Apr;59(4):1233-45.

14 Rosebraugh MR, Widness JA, Nalbant D, Veng-Pedersen P. A mathematical modeling approach to quantify the role of phlebotomy losses and need for transfusions in neonatal anemia. Transfusion. 2013 Jun;53(6):135360.

15 Shannon KM, Keith JF 3rd, Mentzer WC, Ehrenkranz RA, Brown MS, Widness JA, et al. Recombinant human erythropoietin stimulates erythropoiesis and reduces erythrocyte transfusions in very low birth weight preterm infants. Pediatrics. 1995 Jan;95(1):1-8.

16 Richardson DK, Gray JE, McCormick MC, Workman K, Goldmann DA. Score for Neonatal Acute Physiology: a physiologic severity index for neonatal intensive care. Pediatrics. 1993 Mar;91(3):617-23.

17 Kling PJ, Sullivan TM, Leftwich ME, Roe DJ. Score for neonatal acute physiology and phlebotomy blood loss predict erythrocyte transfusions in premature infants. Arch Pediatr Adolesc Med. 1997 Jan;151(1):27-31.

18 Bednarek FJ, Weisberger S, Richardson DK, Frantz ID 3rd, Shah B, Rubin LP; SNAP II Study Group. Variations in blood transfusions among newborn intensive care units. J Pediatr. 1998 Nov;133(5):601-7.

19 Obladen M, Sachsenweger M, Stahnke M. Blood sampling in very low birth weight infants receiving different levels of intensive care. Eur J Pediatr. 1988 May;147(4):399-404.
20 Widness JA, Madan A, Grindeanu LA, Zimmerman MB, Wong DK, Stevenson DK. Reduction in red blood cell transfusions among preterm infants: results of a randomized trial with an in-line blood gas and chemistry monitor. Pediatrics. 2005 May;115(5):1299-306.

21 Duerden EG, Grunau RE, Guo T, Foong J, Pearson A, Au-Young S, et al. Early procedural pain is associated with regionally-specific alterations in thalamic development in preterm neonates. J Neurosci. 2018 Jan;38(4): 878-86.

22 Tortora D, Severino M, Di Biase C, Malova M, Parodi A, Minghetti D, et al. Early pain exposure influences functional brain connectivity in very preterm neonates. Front Neurosci. 2019 Aug; 13:899.

23 Yeh DD. A clinician's perspective on laboratory utilization management. Clin Chim Acta. 2014 Jan;427:145-50.

24 Lippi G, Fostini R, Guidi GC. Quality improvement in laboratory medicine: extra-analytical issues [vii.]. Clin Lab Med. 2008 Jun; 28(2):285-94.

25 United States Centers for Disease Control and Prevention. Guidelines for the Prevention of Intravascular Catheter-Related Infections, 2011. Available from: https://www.cdc.gov/ hai/pdfs/bsi-guidelines-2011.pdf.

26 Boo NY, Wong NC, Zulkifli SS, Lye MS. Risk factors associated with umbilical vascular catheter-associated thrombosis in newborn infants. J Paediatr Child Health. 1999 Oct; 35(5):460-5

27 Fletcher MA, Brown DR, Landers S, Seguin J. Umbilical arterial catheter use: report of an audit conducted by the Study Group for Complications of Perinatal Care. Am J Perinatol. 1994 Mar;11(2):94-9.

28 Oelberg DG, Baker A, Quast D, Worley L. Impact of umbilical catheterization on morbidity and mortality in extremely premature newborns. J Neonatal Perinatal Med. 2014; 7(1):13-9.

29 Counsilman CE, Heeger LE, Tan R, Bekker V, Zwaginga JJ, Te Pas AB, et al. Iatrogenic blood loss in extreme preterm infants due to frequent laboratory tests and procedures. J Matern Fetal Neonatal Med. 2019 Oct 6;1-6. 